American Journal of Applied Sciences 5 (5): 559-564, 2008

ISSN 1546-9239

(C) 2008 Science Publications

\title{
Optimization of Electric Power Distribution Using Hybrid Simulated Annealing Approach
}

\author{
${ }^{1}$ Walid Ahmed and ${ }^{2}$ Alaa F. Sheta \\ ${ }^{1}$ Technical and Vocational Institute, Arab Academy for Science, \\ Technology and Maritime Transport, Alexandria, Egypt \\ ${ }^{2}$ Department of Information Technology, Al-Balqa Applied University, Salt, Jordan
}

\begin{abstract}
The key goal of electric power distribution companies is to provide a high quality of service with a low cost of operation. The growing customer needs requires a re-distribution of the Power over various nodes of the Distributed Generation (DG) facilitates. The re-distribution might cause over load on various parts of the networks which if not correctly optimized might increase the cost of maintenance and affect the overall network reliability. This is why it is urgently requited to find a methodology that can effectively provide a schema for re-distribution of the power and achieve both customers and power companies contracting objectives. In this paper, we explore our new proposed idea of using a simulated annealing based local search technique to provide an efficient power load distribution for distributed generation network. On doing this, we will apply our approach on the famous IEEE14 and IEEE30 power systems as two test cases. The developed results show the significant of the proposed approach.
\end{abstract}

Key words: Simulated annealing, dispersed generation, electric power distribution

\section{INTRODUCTION}

Distributed Generation (DG) attracted much interest as a way to cost-effectively achieve additional capacity. Traditional techniques for planning on power distribution are often scarce for defining the optimal economical way to use the DG options and not to move a step ahead by going for network or stations upgrades. It is urgently needed to find innovative planning methods and efficient computer software systems to help to better understand the exact economic impact for various options ${ }^{[1,2,3,4]}$. There are several benefits for employing a distributed generation optimization techniques. They include:1) providing a standby/backup power to improve availability and reliability of electric power system; 2) allow peak load shaving; 3) advertise power back to utilities or other users; 4) provide a quality improvement to power use; 5) help in reducing the total power loss within the power network ${ }^{[5]}$ Major issues concern about the interconnection of DG includes protection, power quality, voltage regulation and size if injected power were explored in ${ }^{[6]}$. The issue of Reliability modeling of distributed generation in conventional distribution systems planning and analysis was explored $\mathrm{in}^{[7]}$. Authors presented a reliability model for solving the distributed generation planning problem in the new competitive environment ${ }^{[7] .}$. It was shown that the utility companies must increasing the market value of the services by providing the efficient amount of reliability and mean while, lowers its costs for operation, maintenance and construction of new facilities in order to provide lower rates for customers. Many authors reported results on applying Genetic Algorithms (GAs) to solve the power system planning problems. $\mathrm{In}^{[8]}$, authors provided a new technique based genetic algorithms to optimize the sizing and provide a better way to use the distributed generation resources in a 43 and 93 buses MV distribution networks. The applicability of optimizing the distribution of power based simulated annealing technique to the analysis of power systems was provided in ${ }^{[9]}$. The basic idea of using simulated annealing and applications to unit commitment, maintenance scheduling and reactive power sources planning were also presented. In many applications, it was observed that genetic algorithms can suitably be applied to handle the power distribution and planning problems if they are collectively used with other techniques such as simulated annealing, tabu search, fuzzy sets, etc ${ }^{[10,11]}$. The use of hybrid Genetic Algorithm (GA) and Simulated Annealing (SA) approach for the scheduling of generator maintenance in power systems using an integer representation were discussed in ${ }^{[12]}$. Author provided a case study depends on formulating the optimization problem as an integer

Corresponding Author: Walid Ahmed, Technical and Vocational Institute, Arab Academy for Science,

Technology and Maritime Transport, Alexandria, Egypt 


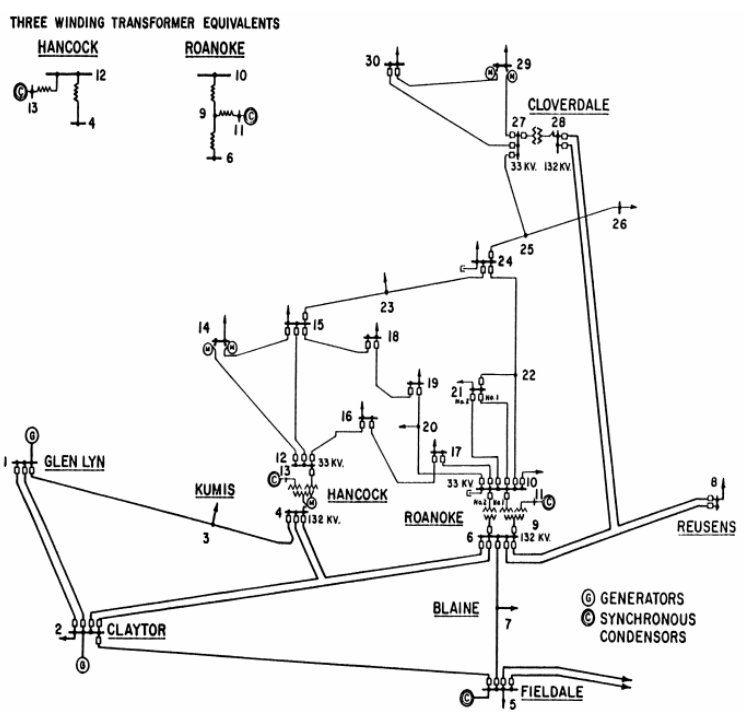

Fig. 1 IEEE 14 bus power system ${ }^{[13]}$

programming problem using a reliability-based objective function to satisfy problem constraints. They claim that the developed results using the hybrid approach is better than using only genetic algorithms or only on simulated annealing.

Our goal in this paper is to explore the use of a hybrid simulated annealing approach to find the optimal amount of electrical power which needs to be injected at specific nodes on a distributed power system. We will test our proposed approach on the IEEE14 and IEEE30 power systems under study. In Fig. 1 and 2, we show the two power generation systems. The better the selected values of the injected power, the better will be the performance of the power system. This will lead to smaller amount amounts of power losses.

Problem formulation: The problem of DG of power system load represents a challenge for both the designer and the system analyst ${ }^{[13,14]}$. This problem involves solving number of equations. These equations include the a.c load flow equations which are used to carry out an accurate load flow analysis and help in providing a complete distribution of the active and reactive power. The constraints equations also include the node active power equation, the line flow equation and limitation of variable constraints. These equations provide a strong system non-linearity. Non linear power flow equations can be described as follows:-

$$
\mathrm{P}_{\mathrm{K}}=\mathrm{V}_{\mathrm{K}} \sum_{\mathrm{n}=1}^{\mathrm{N}} \mathrm{V}_{\mathrm{kn}} \mathrm{V}_{\mathrm{n}} \operatorname{Cos}\left(\delta_{\mathrm{k}}-\delta_{\mathrm{n}}-\theta_{\mathrm{kn}}\right)
$$

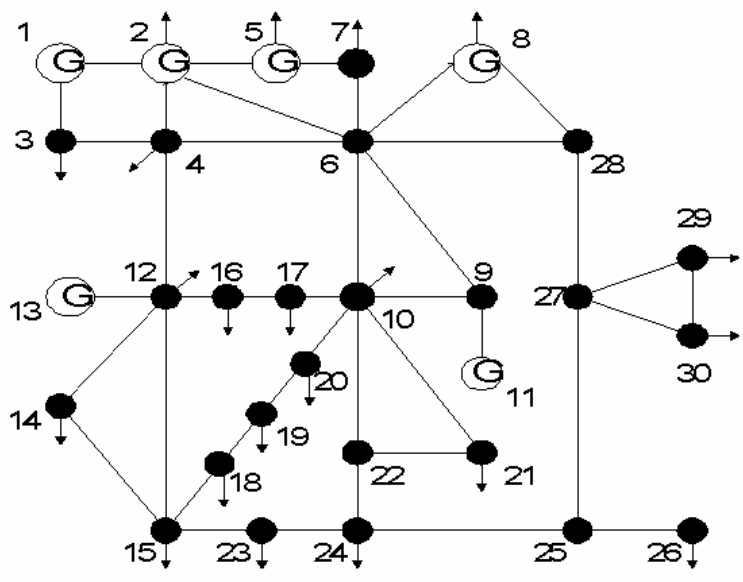

Fig. 2: IEEE 30 bus power system ${ }^{[14]}$

$$
\mathrm{Q}_{\mathrm{K}}=\mathrm{V}_{\mathrm{K}} \sum_{\mathrm{n}=1}^{\mathrm{N}} \mathrm{V}_{\mathrm{kn}} \mathrm{V}_{\mathrm{n}} \operatorname{Sin}\left(\delta_{\mathrm{k}}-\delta_{\mathrm{n}}-\theta_{\mathrm{kn}}\right)
$$

where: $P_{k}, Q_{k}, V_{k}, \delta_{k}$ are real power, reactive power, voltage and phase angle for bus $\mathrm{k} . \mathrm{V}_{\mathrm{kn}}, \theta_{\mathrm{kn}}$ are the admittance between bus $\mathrm{k}$ and $\mathrm{n}$ with the phase angle $\theta . \mathrm{n} \in\{1, \ldots, \mathrm{N}\} . \mathrm{N}$ is the number of busses ${ }^{[15]}$.

Simulated Annealing (SA) algorithm: SA is motivated by an analogy to annealing in solids. The algorithm starts from a valid solution and randomly generates new states for the problem and calculates the associated cost function. Simulation of the annealing process starts at high empirical temperature. A new state is randomly chosen and the difference in cost function is calculated ${ }^{[16]}$. The probability of accepting a worse solution will be dependent on the current temperature. SA aims to remove the drawback of hill climbing search technique ${ }^{[17]}$.

Hybrid SA approach: The proposed search approach is a combination of simulated annealing and neighborhood search. The search space is mapped using integer values representing steps. The search starts with the middle value of a step for each node. If during the neighborhood search a new solution is detected, either because it is directly better or because it is worse but still accepted probabilistically, the neighborhood search stops at the current temperature and a new temperature is taken.

The number of iterations at a certain temperature is not fixed, if a better solution is found. An instance 
decrement in temperature is executed if a better solution is found. The number of iterations at a certain temperature is not allowed to exceed a maximum number which is equivalent to the neighborhood size. The fact that the neighborhood search stops when finding a better solution, makes our neighborhood search resembles the Hill Climbing Technique in a better way as a worse solution that might contain an optimum subpart or might drive the search towards an optimum solution might still be selected to be the current solution. The proposed hybrid search technique differs from the standard simulated annealing since it is decrementing the temperature a full neighborhoods search is applied.

Problem coding: The solution in our case consists of a numbers of subparts. Since we are concerned about determining the amount of power to be injected by different embedded generators we made the number of subparts equal to the number of generators embedded in the power network. The power to be added at certain node in a bus $i$ is given as follows:

$$
\mathrm{P}_{\mathrm{i}}=\operatorname{MinP}+\left(\frac{\text { step }_{\mathrm{i}}}{\mathrm{M}} * \mathrm{MaxP}\right)
$$

$\mathrm{M}$ is the maximum steps; MaxP is the maximum power to be added per node; MinP is the minimum power to be added per node.

Coding the solutions into steps with fixed range will force all possible searched solutions not to violate constrain $\mathrm{C} 1$. If we assume that the number of generators to be embedded equals four, we can decode the DG problem parameters as (a,b,c,d).

$a, b, c$ and $d$ represent the required values of the power to be injected. This simple way of encoding the problem solution allows more flexibility to add or remove nodes for injection to the distributed power system. In general, we can summaries the steps of the proposed algorithms as follows: 1) Start with an initial solution where each subpart will have steps equal 0.5 M; 2) Apply Neighborhood search procedure; 3) Decrement Temperature 4) If final temperature reached then end. If not reached go to step 2.

Objective function and constraints handling: The mathematical equation which describes the objective function we aim to minimize is as follows:

$$
\text { Objected Function }=\operatorname{Min} \sum_{i=1}^{n} P_{i}
$$

$\mathrm{P}_{\mathrm{i}}$ is defined as the amount of power injected to a node at bus $i$ and $n$ is the total number of buses in the power system. The objective function reflects directly the power losses, since the summation in Eq. (4) are implemented using the load power with the negative sign. The Newton-Raphson method ${ }^{[18]}$ was presented as a solution for the above problem. The DG problem, in our case, has two constrains. They are:

Constrain 1 (C1): The amount of added power per node to be within a certain specified range.

$$
\forall_{\mathrm{i}} \operatorname{Min} \mathrm{P} \leq \mathrm{P}_{\mathrm{i}} \leq \operatorname{Max} \mathrm{P}
$$

MinP, MaxP are the power limits for power injected at node $\mathrm{i}$.

Constrain 2 (C2): The total amount of added power to all nodes to be within a certain specified range.

$$
\mathrm{m}_{1} \leq \sum_{\mathrm{i}=\mathrm{j}}^{\mathrm{k}} \mathrm{p}_{\mathrm{i}} \leq \mathrm{m}_{2}
$$

$\mathrm{i} \in\{\mathrm{j}, \ldots, \mathrm{k}\}$ is the voltage controlled bus's number. And, $\mathrm{m}_{1}, \mathrm{~m}_{2}$ are the power to be injected as power limits. Each time a solution is considered as a candidate, power flow calculations are executed to calculate the objective function value of this candidate solution. The procedure which describes our neighborhood search algorithm is shown as follows:

Procedure Neighborhood Search (Individual, T)

Begin

New_indiv = Individual;

$\mathrm{f1}$ = fitness_of_Individual

for $\mathrm{i}=1$ to $\mathrm{k}$; ( $\mathrm{k}$ maximum number of iterations)

$\mathrm{r} 1=$ random number in $[-\mathrm{p}, \mathrm{p}] ; ;$ ( $\mathrm{p}$ arbitrary chosen $)$

r2 = random integer number in[1, no. of power nodes];

Add $r 1$ to the power of node $r 2$ of New_indiv without violating $\mathrm{C} 1, \mathrm{C} 2$

$\mathrm{f} 2$ = fitness of New_indiv; If (f2>f1) Individual = new individual; Break;

Rnd: Random number in $[0,1]$; If $\left(\mathrm{P}_{\mathrm{r}}\right)$ Individual = new individual; Break

End

End 
Am. J. Applied Sci., 5 (5): 559-564, 2008

Table 1: Tuning parameters for the hybrid approach in both cases

\begin{tabular}{ll}
\hline \multirow{2}{*}{ Problem } & IEEE14 \\
Maximum number of iterations & IEEE30 \\
Initial Temperature & 50 \\
Final Temperature & 100 \\
Cooling Schedule & 0 \\
& $\mathrm{~T}=\mathrm{T}-1$ \\
& $\mathrm{~T}=\mathrm{T}-1$, if $\mathrm{T}<=50$ \\
Acceptance Function & $\mathrm{T}=\mathrm{T}-0.2$, if $50<\mathrm{T}<100$ \\
& $\mathrm{Pr}_{\mathrm{r}:\left(\mathrm{e}^{-90 / \mathrm{T}}\right)>\mathrm{r}}$ \\
& $\mathrm{Pr}_{\mathrm{r}:\left(\mathrm{e}^{-20 / \mathrm{T}}\right)>\mathrm{r}}$ \\
Generating Function & $\mathrm{g}\left(\Delta_{\mathrm{x}}, \mathrm{T}\right)=\frac{1}{\sqrt{2 \pi \mathrm{T}}} \mathrm{e}^{\frac{-\Delta \mathrm{x}^{2}}{2^{\mathrm{T}}}}$ \\
\hline
\end{tabular}

Experimental results: To show the advantages of the proposed algorithm, two case studies were considered. They are the IEEE14 and IEEE30 systems. The IEEE14 and IEEE30 Bus are two well known systems. The former consists of 14 power bus and 20 power lines and the later consists of 30 power bus and 41 power links. The proposed Hybrid SA approach was implemented using MATLAB. According to the experience we developed for the IEEE14, it was found that the points where dispersed generation units are chosen were buses $2,3,6,8$. These buses are voltage controlled. In the case of the IEEE30 bus, we choose to put our dispersed generation units at buses $2,5,8,11,13$. These buses are also voltage controlled ones.

The setting parameters of the Hybrid SA approach are given, for the two systems, in Table 1. The search space of the IEEE30 system found to be relatively larger than that of the IEEE14 system. The cooling schedule used for the IEEE 30 was slower than that the IEEE 14 system. The values of steps were selected from 0 to 100 (i.e., $M=100$ ). The values of the $\mathrm{MinP}$ and MaxP were 0 p.u. and 1.5 p.u, respectively. The total amount of power to be added at all nodes should be between 1.5-2.5 p.u.

\section{RESULTS AND DISCUSSION}

In is clear that the proposed hybrid algorithm recommend that there is no need to add power at bus 2 since both systems under study already have power units at this bus. It was also found that the differences in power losses are not significant in the acceptance function value. This is why a constant empirical value was chosen. The run time increased with the increase in size of the power network.
Table 2: The output results for the IEEE 14 and the IEEE 30 power systems

\begin{tabular}{lll}
\hline Problem & IEEE14 & IEEE30 \\
\hline Run Time in minutes & 2 & 19 \\
Temperature for best Individual & Temp $=10^{\circ}$ & Temp $=4^{\circ}$ \\
Temperature when program stopped & Temp $=1^{\circ}$ & Temp $=1^{\circ}$ \\
Minimum Losses (in p.u.) & 0.1491 & 0.0242 \\
Power added at node 2 & 0.0 & 0.0 \\
Power added at node 3 & 1.005 & 0.990 \\
Power added at node 6 & 0.630 & 0.405 \\
Power added at node 8 & 0.525 & 0.585 \\
Sum of added power & 2.16 & 0.330 \\
\hline
\end{tabular}

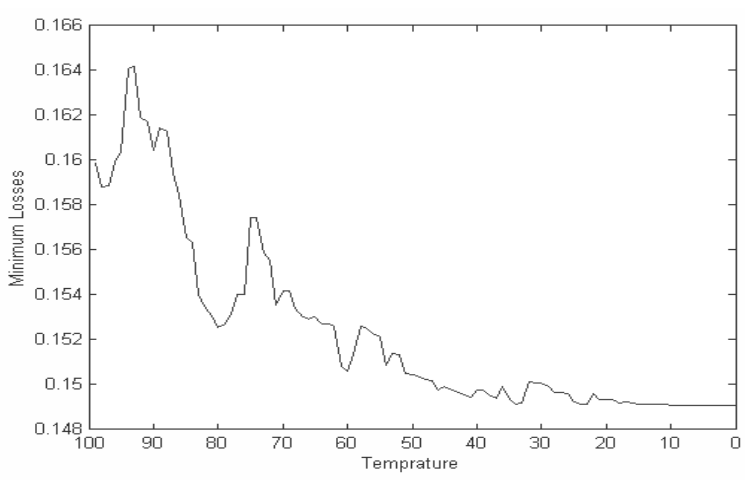

Fig. 3: Minimum Losses versus Temperature using Hybrid SA approach for the IEEE14 system

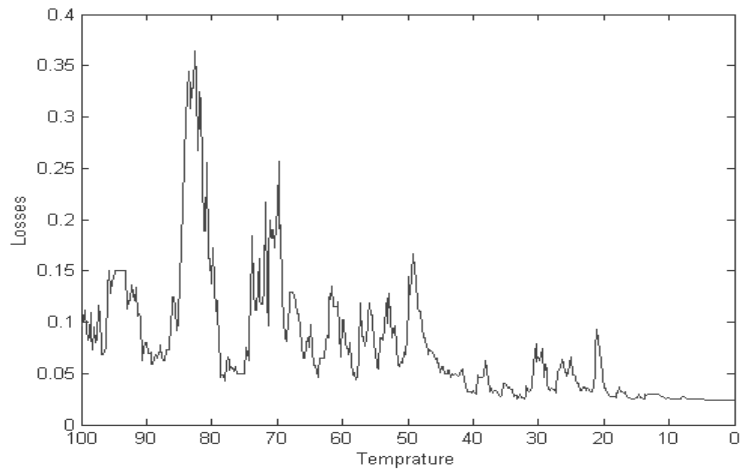

Fig. 4: Minimum Losses versus Temperature using Hybrid SA approach for the IEEE30 system

The above results are fully described in Table 2 . Figure 3 and 4 show the behaviors of power losses with the continuous degree of temperature using proposed algorithm for both IEEE14 and IEEE30 systems. It is clear from figures that the hybrid SA approach provided less power losses than traditional approaches. A complete flow chart of the Hybrid SA approach is shown in Fig. 5. 


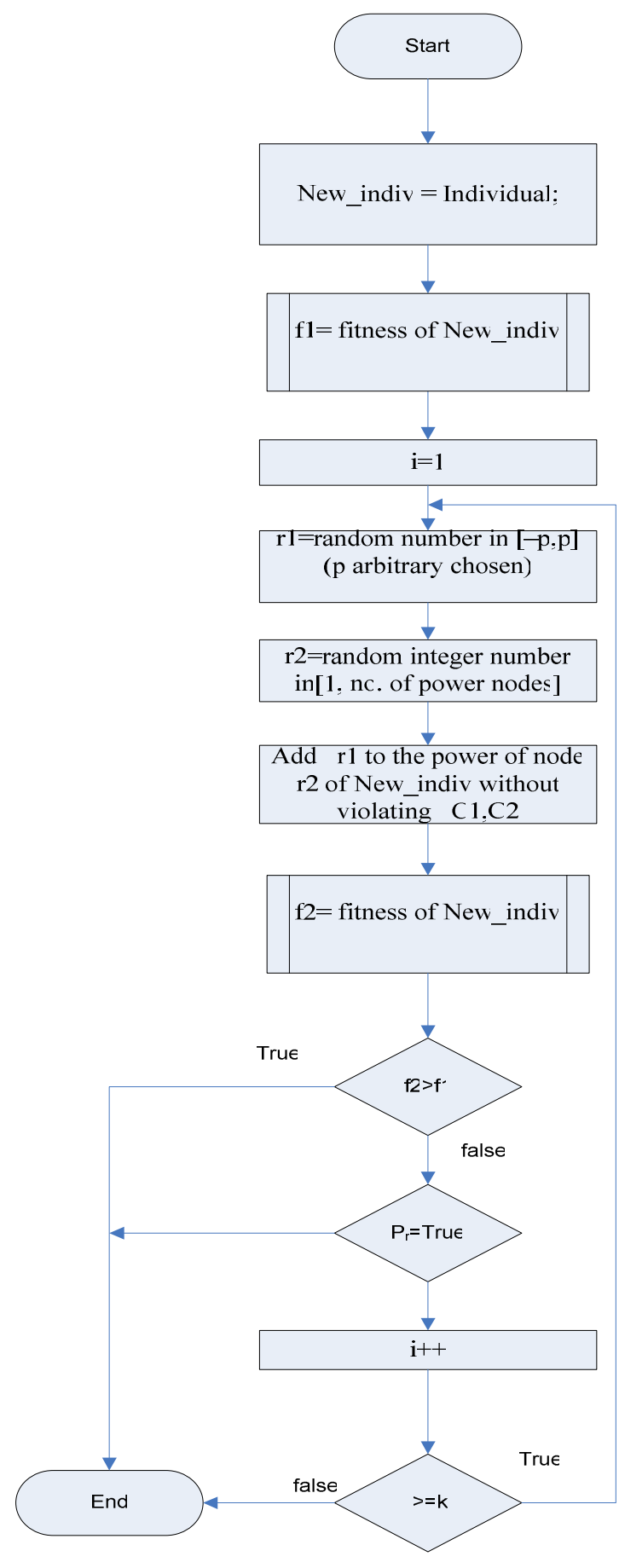

Fig. 5: Hybrid SA Search Approach

\section{CONCLUSION}

In this study, we proposed a Hybrid Simulated Annealing approach. The goal of the approach was to use this algorithm to help is solving the problem of optimizing the exact amount of electrical power to be injected in a distributed generation system. Achieving this goal will help in providing an efficient power generation planning, reducing the total power loss and satisfying number of design constrains. Two case studies were provided. The results show that the Hybrid SA proposed approach can handle this difficult optimization problem with promising results.

\section{REFERENCES}

1. Dugan, R.C., T.E. McDermott and G.J. Ball, 2000. Distribution planning for distributed generation. Rural Electric Power Conference, pp: 1-7.

2. Rackliffe, G., 2000. Guidelines for planning distributed generation systems. IEEE Power Eng. Soc. Summer Meeting, 3: 1666-1667.

3. Pereira, J., J.T. Saraiva and M.T. Ponce de Leao, 1999. Identification of operation strategies of distribution networks using a simulated annealing approach. Int. Conference on Electric Power Eng., pp: 42.

4. Dugan, R.C., T.E. McDermott and G.J. Ball, 2001. Planning for distributed generation. IEEE Ind. Applications Magazine, 2: 1077-2618.

5. Naihu, L., X. Yan and H. Chen, 2000. FACTSbased Power Flow Control in Interconnected Power System. IEEE Trans. Power Syst., 15: 257-262.

6. Kroposki, B. and S. Englbreston, 2003. Validation of IEEE P1547.1 Interconnection test proceduresASCO7000 Soft Load Transfer System, NREL/TP-560-34870.

7. Chowdhury, A.A., S.K. Agarwal and D.O. Koval, 2002. Reliability modeling of distributed generation in conventional distribution systems planning and analysis. 37th IAS Annual Meeting Industry Applications Conference, 2: 1089-1094.

8. Silvestri, A., A. Berizzi and S. Buonanno, 1999. Distributed generation planning using genetic algorithms. Int. Conference on Electric Power Eng., pp: 257.

9. Deeb, N., 1992. Simulated annealing in power systems. IEEE Int. Conference Syst. Man Cybernetics, 2: 1086-1089.

10. Ahmed, R. Abdelaziz and Walid Ali, 2003. Dispersed Generation Planning Using A New Evolutionary Approach. Proceedings of the IEEE 2003 Bologna Power Tech. Conference, June 2326, Bologna, Italy. 
11. Nara, K., 1997. Genetic algorithm for power systems planning. 4th International Conference on Advances in Power System Control, Operation and Management, 1: 60-65.

12. Dahal, K.P., G.M. Burt, J.R. McDonald and S.J. Galloway, 2000. GA/SA-based hybrid techniques for the scheduling of generator maintenance in power systems. Proceedings of the 2000 Congress on Evolutionary Computation, 1: $567-574$.

13. Bretas, N.G., J.B.A.Jr. London, L.F.C. Alberto and A.S. Bretas, 2005. A topological approach to the identification of critical measurements in powersystem state estimation. IEEE Trans. Circuits Syst., 52: 139-147.

14. Jayaweera, D., S. Galloway, G. Burt and J.R. McDonald, 2007. A Sampling Approach for Intentional Islanding of Distributed Generation. IEEE Trans. Power Syst., 22: 514-521.
15. Duncan, J., Glover, Mulukutla and S. Sarma, 2001. Power System Analysis and Design, Publisher: Thomson-Engineering. 3rd Edn.

16. Negnevitsky, M., 2005. Artificial Intelligence: A Guide to Intelligent Systems. Addison Wesley.

17. Ingber, K.L., 1993. Simulated annealing: Practice versus theory. Mathematical and Computer Modeling, 18: 29-57.

18. Abdel-Rahim, A.M.M. and N.P. Padhy, 2004. Newton-Raphson UPFC model for power flow solution of practical power networks with sparse techniques. Proceedings of the 2004 IEEE International Conference on Electric Utility Deregulation. Restructuring and Power Technol., 1: 77-83. 